\title{
Análisis Genómico de Mannheimia haemolytica Serotipo A2 para la Identificación de Potenciales Candidatos Vacunales contra la Neumonía en Alpacas
}

\author{
Whole-Genome Analysis of Mannheimia haemolytica Serotype A2 to Identify \\ Potential Vaccine Candidates against Acute Pneumonia in Alpacas
}

\author{
Eduardo Juscamayta L.,3, Lenin Maturrano H., ${ }^{1,2}$, Raúl Rosadio A. ${ }^{1}$
}

\section{Resumen}

El objetivo del presente trabajo fue identificar antígenos inmunoprotectivos en la secuencia genómica de Mannheimia haemolytica serotipo A2 Ovino, aislado de ovino y disponible en las bases de datos, que puedan ser utilizados como potenciales candidatos vacunales contra la neumonía en alpacas. Para ello, se empleó la secuencia completa del genoma de este microorganismo disponible en las bases de datos y mediante el uso de herramientas bioinformáticas se procedió a la búsqueda e identificación de genes candidatos, la anotación funcional, la predicción de la localización subcelular y la identificación de motivos de secuencias, incluyendo dominios transmembrana, peptidos señal de secreción y lipoproteínas. Los factores de virulencia fueron identificados en la base de datos de factores de virulencia (VFDB) y en la base de datos microbiana de virulencia (MvirDB). Los criterios de selección incluyeron proteínas conservadas, secretadas y asociadas a superficie con hélices transmembrana $\leq 1$. Se realizó la búsqueda de homología de genes con la base de datos de antígenos protectivos (Protegen), permitiendo la identificación de 23 antígenos potencialmente inmunoprotectivos, conservados entre los serotipos virulentos de $M$. haemolytica y otros patógenos de la familia Pasteurellaceae. Los genes identificados están relacionados con la patogénesis, virulencia y evasión del sistema immune del hospedero y podrían ser grandes candidatos a ser evaluados como potenciales vacunas contra la pasteurelosis neumónica.

Palabras clave: Mannheimia haemolytica, pasteurelosis neumónica, vacunología reversa, factores de virulencia, candidatos vacunales inmunoprotectivos

\footnotetext{
${ }^{1}$ Sección de Biología y Genética Molecular, Laboratorio de Microbiología y Parasitología Veterinaria, ${ }^{2}$ Laboratorio de Zootecnia y Producción Agropecuaria, Facultad de Medicina Veterinaria, Universidad Nacional Mayor de San Marcos, Lima, Perú

${ }^{3}$ E-mail: ejuscamaytal@gmail.com
}

Recibido: 26 de agosto de 2016

Aceptado para publicación: 29 de enero de 2017 
The aim of this study was to identify immunoprotective antigens in the genomic sequence of Mannheimia haemolytica serotype A2 isolated from sheep and available in databases that can be used as potential vaccine candidates against pneumonia in alpacas. To do this, the complete genome sequence of this organism available in databases was used and by using bioinformatics tools was proceeded to search for and identification of candidate genes, functional annotation, predicting the subcellular localization and identification of sequence motifs including transmembrane domains, secretion signal peptides and lipoproteins. Virulence factors were identified in the database of virulence factors (VFDB) and the microbial database of virulence factors (MvirDB). Selection criteria included conserved proteins, secreted and associated surface with transmembrane helices $\leq 1$. Homology search of genes was performed with the database of protective antigens (Protegen), allowing the identification of 23 potentially immunoprotective antigens conserved between virulent $M$. haemolytica serotypes and other pathogens of the family Pasteurellaceae. The genes identified are related to the pathogenesis, virulence and immune system evasion of the host and they could be great candidates to be evaluated as potential vaccines against pneumonic pasteurellosis.

Key words: Mannheimia haemolytica, pneumonic pasteurellosis, reverse vaccinology, virulence factors, vaccine candidates, immunoprotective

\section{INTRODUCCIÓN}

Mannheimia haemolytica es una bacteria Gram-negativa, perteneciente a la familia Pasteurellaceae, y principal agente etiológico de la neumonía o pasteurelosis neumónica en bovinos y ovinos. Esta bacteria causa grandes pérdidas económicas en la industria ganadera a nivel mundial (Mohamed y Abdelsalam, 2008; Lawrence et al., 2010), y ha sido asociada, junto con Pasteurella multocida, a casos de neumonía en camélidos sudamericanos domésticos, siendo la segunda causa más importante de mortalidad de alpacas en Perú (Rosadio et al., 2011).

Mannheimia haemolytica comprende 12 serotipos capsulares (Angen et al., 1999), siendo A 1 y A6 los serotipos más aislados en casos de procesos neumónicos en bovinos (Davies et al., 2001), en tanto que A2 es en ovinos (Saadati et al., 1997), aunque también se observa un incremento en la prevalencia de los serotipos A5, A6 y A7 (Ewers et al., 2004); sin embargo, se desconoce los serotipos asociados a los casos neumónicos en crías de alpacas.

El uso extensivo de antibióticos como terapia contra la neumonía ha incrementado la incidencia de multidrogoresistencia de $M$. haemolytica (Kehrenberg et al., 2001), de allí que el control se ha enfocado hacia el desarrollo de vacunas a partir de la identificación de los factores de virulencia, especialmente en bovinos (Zecchinon et al., 2005). Se dispone de vacunas comerciales contra pasteurelosis neumónica para bovinos y ovinos; sin embargo, solo inducen protección a corto plazo contra la infección y no proveen una inmunidad protectiva cruzada contra serotipos heterólogos de $M$. haemolytica (Lacasta et al., 2015). La falta de vacunas efectivas radica principalmente en la multiplicidad de serotipos de esta bacteria (Gonzalez et al., 2013), las diferencias genéticas y la pobre inmunogenicidad entre los factores de virulencia de cepas de un mismo serotipo (Davies et al., 1997; Davies y Baillie, 2003). 
No se dispone actualmente del secuenciamiento del genoma de $M$. haemolytica aislado de alpacas; no obstante, el secuenciamiento del serotipo A1 (Gioia et al., 2006) y del serotipo A2 (Lawrence et al., 2009), aislados de bovinos y ovinos, respectivamente, ofrecen una nueva estrategia para la identificación de candidatos vacunales, denominada vacunología reversa, que ha permitido el desarrollo de vacunas efectivas, mediante la predicción de potenciales antígenos utilizando herramientas bioinformáticas (Bambini y Rappuoli, 2009).

El presente estudio se basa en el enfoque genómico de la vacunología reversa para identificar potenciales factores de virulencia conservados e inmunoprotectivos utilizando el genoma de $M$. haemolytica serotipo A2 Ovino disponible en la base de datos del NCBI (Número de accesión: ACZX01000000). Se parte de la base de que existe una crianza mixta de alpacas y ovinos, y que las herramientas de la vacunología reversa permiten identificar candidatos vacunales comunes a varios serotipos. El objetivo del presente estudio fue identificar dichos candidatos vacunales en un genoma disponible en la base de datos que puedan ser empleados en el desarrollo futuro de vacunas de tercera generación contra la pasteurelosis neumónica de las alpacas.

\section{Materiales y Métodos}

Para la identificación de regiones codificantes del genoma de $M$. haemolytica serotipo A2 Ovino y poder seleccionar potenciales candidatos vacunales, se diseñó un in-house pipeline semiautomático usando programas bioinformáticos y scripts en Perl (Stajich et al., 2002) (Figura 1). El análisis y filtro de la información generada fue basada en una serie de criterios según el enfoque genómico de la vacunología reversa.

\section{Anotación Estructural y Funcional}

Se utilizó la secuencia del genoma borrador de M. haemolytica A2 Ovino, disponible en la base de datos del National Center of Biotechnology Institute (NCBI) (Número de accesión: ACZX00000000). El genoma fue obtenido en formato FASTA y en 144 contigs. Los contigs fueron concatenados en un solo archivo multifasta, utilizando el programa CAP3 (Huang y Madan, 1999).

Las secuencias codificantes (CDS) fueron identificadas en los mismos contigs utilizando el conjunto de programas pertenecientes a Glimmer 3.0.2 (Delcher et al., 2007). El training set of genes fue obtenido a partir de todos los contigs del genoma, con el programa long-orfs, y la lista de coordenadas de secuencias fueron extraídas con el programa extract. Los training set of genes fueron utilizados para construir el Modelo de Contexto Interpolado (ICM), mediante el programa build-ICM. Este modelo fue finalmente utilizado en el programa Glimmer para la identificación de genes presuntivamente codificantes de proteínas, considerando al genoma de tipo lineal por estar en contigs. Asimismo, un script en Perl fue utilizado para adicionar a la lista de coordenadas de los marcos abiertos de lectura (ORFs, orf.predict), un campo extra que especifique los contigs específicos donde pertenecen dichas coordenadas, necesarios para ser extraídos mediante el programa multi-extract (orfs.seq).

Previo a la anotación funcional, cada CDS fue traducida a secuencias de aminoácidos (protein.seq), utilizando el programa transeq del paquete EMBOSS (Rice et al., 2000) aplicando el código genético estándar de bacterias. Estos genes potenciales traducidos fueron anotados por una búsqueda local de homología de secuencias contra la base de datos completa de proteínas no redundantes (nr) del NCBI, usando el pro- 


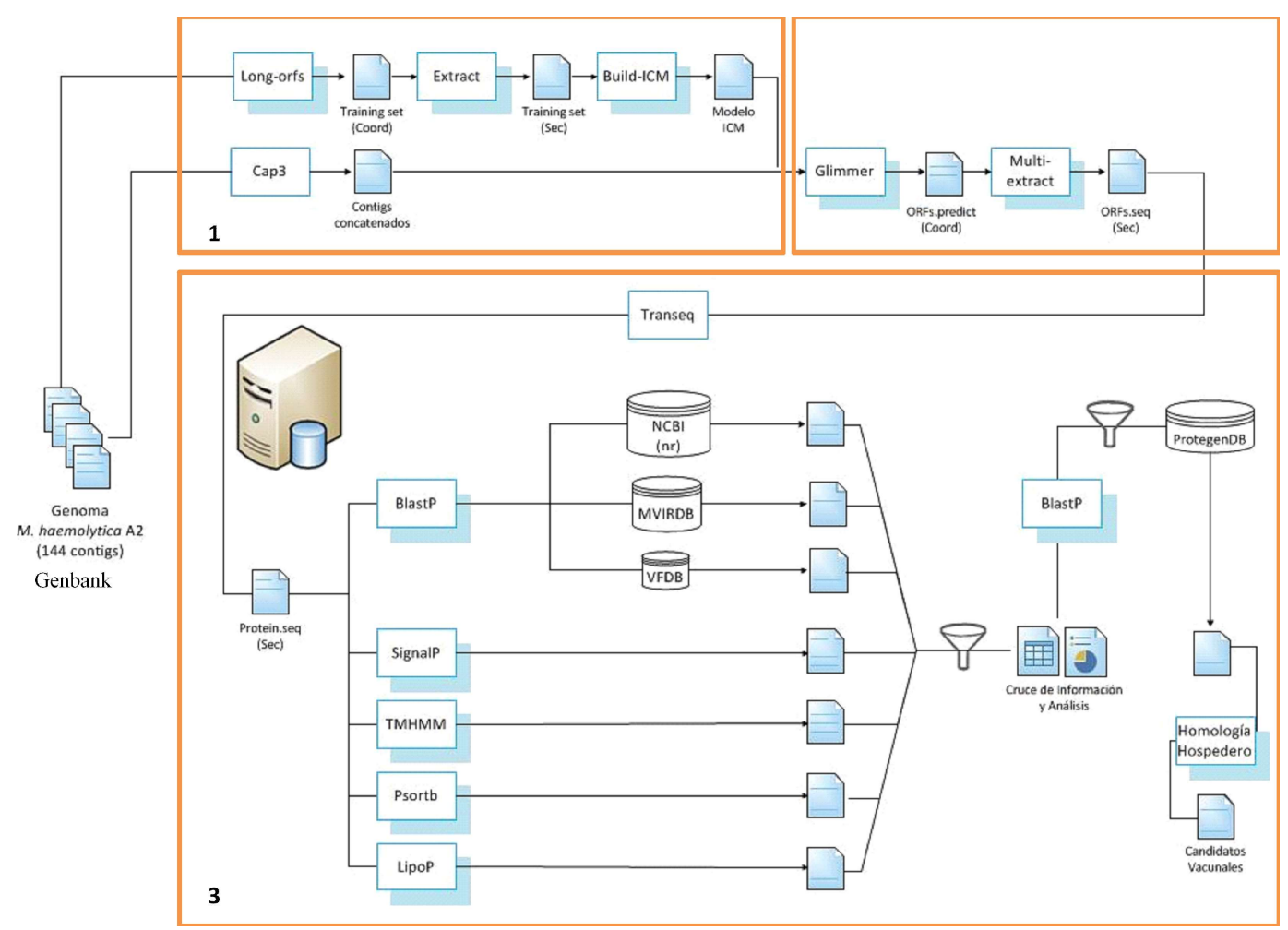

Figura 1. Flujo de datos y subsistemas de la anotación y predicción de candidatos vacunales del genoma de Mannheimia haemolytica serotipo $\mathrm{A}_{2}$ Ovino. Tres subsistemas están representados: (1) Procesamiento del genoma y construcción del modelo; (2) Anotación estructural; (3) Anotación funcional y búsquedas de candidatos vacunales

grama BlastP del paquete BLAST (Altschul et al., 1997), tomando en cuenta una identidad mayor al $70 \%$ sobre el $90 \%$ de longitud (cobertura) y un e-value $\leq 1 \mathrm{e}-10$.

\section{Predicciones}

De factores de virulencia

Las posibles proteínas, homólogas a otras previamente caracterizadas como factores de virulencia en otros organismos, fueron identificadas por análisis de BlastP contra la Base de Datos de Factores de Virulencia (VFDB) (Chen et al., 2016) y la base de datos microbiana de virulencia (MvirDB) (Zhou et al., 2007), respectivamente, consi- derando un e-value $\leq 1 \mathrm{e}-10$ y una identidad $\geq 30 \%$. Las secuencias de los posibles factores de virulencia obtenidas, fueron analizadas y seleccionadas, en función del e-value, identidad, cobertura y en base a publicaciones previas.

De localización subcelular, de hélices transmembrana y péptidos señal de secreción y lipoproteinas

Se utilizó el programa PSORTb (Yu et al., 2010) para predecir la localización subcelular de todas las proteínas codificadas del genoma de $M$. haemolytica A2 Ovino. Además, se usó algoritmos de búsqueda de secuencias específicas para la identificación 
de motivos de secuencia, incluyendo péptidos señal de secreción (SignalP) (Petersen et al., 2011), sitios de clivaje de lipoproteínas (LipoP) (Juncker et al., 2003) y dominios transmembrana para la identificación de proteínas potenciales de membrana (TMHMM) (Krogh et al., 2001).

\section{Análisis de Datos}

El análisis y filtro de la información generada fue basada en una serie de criterios según el enfoque genómico de la vacunología reversa. En general, las proteínas citoplásmáticas y periplásmicas fueron descartadas, considerando únicamente las proteínas con hélices transmembrana $\leq 1$. En este punto, se seleccionaron proteínas con péptidos señal de secreción y lipoproteínas.

Todas las proteínas de localización en la membrana interna fueron eliminadas y se incluyeron manualmente factores de virulencia de localización extracelular y de membrana externa, además de proteína hipotéticas de origen desconocido. Intencionalmente, un pequeño grupo de factores de virulencia de localización citoplasmática y membrana interna fueron incluidos, con el fin de verificar la eficiencia del pipeline de predicción. La selección de todas las proteínas incluidas fue en base a la comparación entre los factores de virulencia identificados y las proteínas anotadas, teniendo como criterio el nivel de conservación entre serotipos virulentos, evalue, identidad, cobertura y publicaciones previas.

Todos los antígenos obtenidos fueron analizados contra la base de datos curada de antígenos protectivos (Protegen) (Yang et al., 2011), para luego realizar una búsqueda de homología con proteínas de ratón. Finalmente, todos los antígenos menores de 150 aminoácidos y mayores de 1000 aminoácidos fueron removidos.

\section{Resultados}

La anotación del genoma de $M$. haemolytica serotipo A2 Ovino resultó en 2656 secuencias codificantes de proteínas (41.1\% G+C) (Cuadro 1), de las cuales 1948 fueron asignados a una función y entre las proteínas hipotéticas, 645 fueron únicas para $M$. haemolytica, 6 para el fago phiMHaA1 y el resto fueron proteínas «hipotéticas» conservadas dentro de la familia Pasteurellaceae, considerando un e-value $\leq \mathrm{e}-10$ y una identidad mayor al 90\% (Cuadro 1).

La mayoría de las proteínas homólogas identificadas (98\%) fueron altamentes conservadas entre las tres cepas de $M$. haemolytica: A1, A2 Bovino y A2 Ovino con una identidad promedio de $99 \%$, incluyendo una gran cantidad de factores de virulencia. El 2\% de proteínas restantes tuvieron homología con secuencias del fago phiMHaA1 ( $100 \%$ de identidad) y otras proteínas pertenecientes a diferentes especies de la familia Pasteurellaceae (73-100\% de identidad) (Figura 2).

Se identificaron 742 posibles factores de virulencia usando la base de datos MvirDB. Por otro lado, se identificaron 295 posibles factores de virulencia usando la base de datos VFDB, representando las proteínas de captación de hierro (24\%), factores de adherencia $(10 \%)$, proteínas antifagocíticas $(7 \%)$, proteínas relacionadas a la regulación (4\%), endotoxinas $(3 \%)$, toxinas $(2 \%)$ y proteasas de IgA1 $(2 \%)$, entre los más abundantes (Figura 3).

Siguiendo el enfoque genómico de la vacunología reversa, se hizo un screening a las 2656 proteínas y se seleccionaron aquellas con localización celular desde la membrana bacterial interna hacia la externa, seguida por predicción de hélices transmembrana, encontrándose 618 proteínas 
Cuadro 1. Características generales del genoma de Mannheimia haemolytica serotipo $\mathrm{A}_{2}$ Ovino

\begin{tabular}{ll}
\hline Característica & $\mathrm{N}^{\circ}$ \\
\hline Total de longitud de contigs $(\mathrm{pb})$ & $2,621,193$ \\
$\mathrm{~N} .{ }^{\circ}$ de contigs & 144 \\
$\% \mathrm{GC}^{1}$ & 41.1 \\
$\mathrm{~N} .{ }^{\circ}$ de secuencias codificantes $(\mathrm{CDS})^{2}$ & 2,656 \\
$\mathrm{~N} .{ }^{\circ}$ de CDS con función asignada (\%) & $1,948(73 \%)$ \\
$\mathrm{N} .{ }^{\circ}$ de CDS hipotéticos únicos para $\mathrm{M}$. haemolytica $(\%)$ & $645(24 \%)$ \\
$\mathrm{N} .{ }^{\circ}$ de CDS de proteínas hipotéticas conservadas de Pasteurellaceae & 11 \\
$\mathrm{~N} .{ }^{\circ}$ de probables factores de virulencia $(\%)^{3}$ & $742(28 \%)$ \\
$\mathrm{N} .{ }^{\circ}$ de probables factores de virulencia $(\%)^{4}$ & $295(11 \%)$ \\
\hline${ }^{1}$ Contenido de guanina y citosina & \\
${ }^{2}$ Secuencia de DNA codificante & \\
${ }^{3}$ Utilizando la base de datos MvirDB & \\
${ }^{4}$ Utilizando la base de datos VFDB &
\end{tabular}

de membrana plasmática y extracelulares. De estas, solo se consideraron las que tenían hélices transmembrana $\leq 1$, obteniéndose 237 proteínas. Posteriormente, se seleccionaron proteínas que tuvieran las secuencias señal de secreción y de lipoproteínas, excluyéndose manualmente todas aquellas con localización en la membrana interna; se adicionaron 38 factores de virulencia conservados entre nuevos e hipotéticos de localización citoplasmática, membrana interna, membrana externa, extracelular y desconocida, para finalmente contar con 75 factores de virulencia potenciales. De estos, solo se eligieron aquellos que tuvieron homología con proteínas de la base de datos Protegen (e-value $\leq 1$ e- 5 y una identidad $\geq 25 \%$, obteniéndose 26 potenciales candidatos vacunales. Ninguno de estos candidatos tuvo homología con alguna proteína del ratón. Finalmente, las proteínas menores que 150 aminoácidos y mayores que 1000 aminoácidos fueron removidas, resultando en 23 potenciales candidatos vacunales, la mayoría de ellos asociados a membrana externa (Figura 4).
La lista de los 23 potenciales candidatos vacunales se muestran en el Cuadro 2.

\section{Discusión}

En la presente investigación se realizó el análisis del genoma de $M$. haemolytica serotipo A2 Ovino con la finalidad de identificar potenciales candidatos vacunales que brinden una protección cruzada y universal contra la pasteurelosis neumónica, teniendo como estrategia el enfoque genómico de la vacunología reversa.

En número de genes identificados (2656) y el \%GC (41.1\%) (Cuadro 1) fueron comparables a los reportados en previas anotaciones del genoma de $M$. haemolytica A1 (2839; 41\%GC) (Gioia et al., 2006) y A2 (2682; 41\%GC) (Lawrence et al., 2009). El $98 \%$ de las proteínas homólogas fueron identificadas entre las tres cepas de $M$. haemolytica: A1 y A2 Bovino y A2 Ovino, lo que sugiere que la mayoría de los genes son 
Cuadro 2. Potenciales candidatos vacunales de Mannheimia haemolytica srt. $\mathrm{A}_{2}$ Ovino identificados usando el enfoque genómico de la vacunología reversa. Todos los candidatos son conservados entre serotipos virulentos de $M$. haemolytica (identidad $>99 \%$ ) y presentan alta similitud con al menos un dominio de factores de virulencia de patógenos relacionados

\begin{tabular}{|c|c|c|}
\hline $\mathrm{ORF}^{1}$ & Candidato vacunal & Localización \\
\hline orf00051 & Proteína de membrana externa & ME \\
\hline orf00191 & Proteína de secreción hemaglutinina FhaC & ME \\
\hline orf00352 & Lipoproteina Plp4 & ME \\
\hline orf00438 & Proteína estructural leucotoxina & $\mathrm{E}$ \\
\hline orf00614 & Receptor sideróforo de membrana externa familia OMR & ME \\
\hline orf00623 & Neuraminidasa & $\mathrm{D}$ \\
\hline orf00685 & Proteína hipotética COI_0728 & $\mathrm{ME}$ \\
\hline orf00799 & Proteína de membrana externa P4 & ME \\
\hline orf01174 & Proteína de membrana externa P1 & ME \\
\hline orf01125 & Proteína de membrana externa D15 & ME \\
\hline orf01126 & Proteína hipotética MHA_0690 & ME \\
\hline orf01326 & Proteína C de utilización hemo-hemopexina & ME \\
\hline orf01521 & Receptor de hierro de membrana externa familia OMR & ME \\
\hline orf01619 & Transglicosilasa de mureína lítica unida a membrana & ME \\
\hline orf01650 & Adhesina autotransportadora & $\mathrm{E}$ \\
\hline orf01882 & Adhesina/autotransportadora familia AT & $\mathrm{D}$ \\
\hline orf01914 & Proteína de membrana externa modificable al calor & ME \\
\hline orf02261 & Proteína de unión a transferrina $B$ & ME \\
\hline orf02262 & Proteína de unión a transferrina A & ME \\
\hline orf02187 & $\begin{array}{l}\text { Receptor HmbR2 de hemoglobina de membrana externa } \\
\text { familia OMR }\end{array}$ & ME \\
\hline orf02324 & Adhesina/autotransportadora putativa & ME \\
\hline orf02368 & Metaloendopeptidasa específico de IgA familia S6 & ME \\
\hline orf02528 & $\begin{array}{l}\text { Lipoproteína de membrana externa asociado a } \\
\text { peptidoglicano }\end{array}$ & ME \\
\hline
\end{tabular}

${ }^{1}$ ORF: marcos abiertos de lectura; ME: membrana externa; E: extracelular; D: desconocido

altamente conservados para los tres aislados (identidad $>90 \%$ ).

La predicción de candidatos vacunales dio como resultado 23 potenciales antígenos inmunoprotectivos (Cuadro 2). Para los efectos del presente estudio se seleccionaron moléculas asociadas a superficie y proteínas secretadas (con peptidos señal), ya que se ha demostrado que estas moléculas son potenciales dianas para inducir una fuerte respuesta inmune celular en el hospedero (Gamberini et al., 2005). Por el contrario, proteínas no superficiales, así como proteí- 


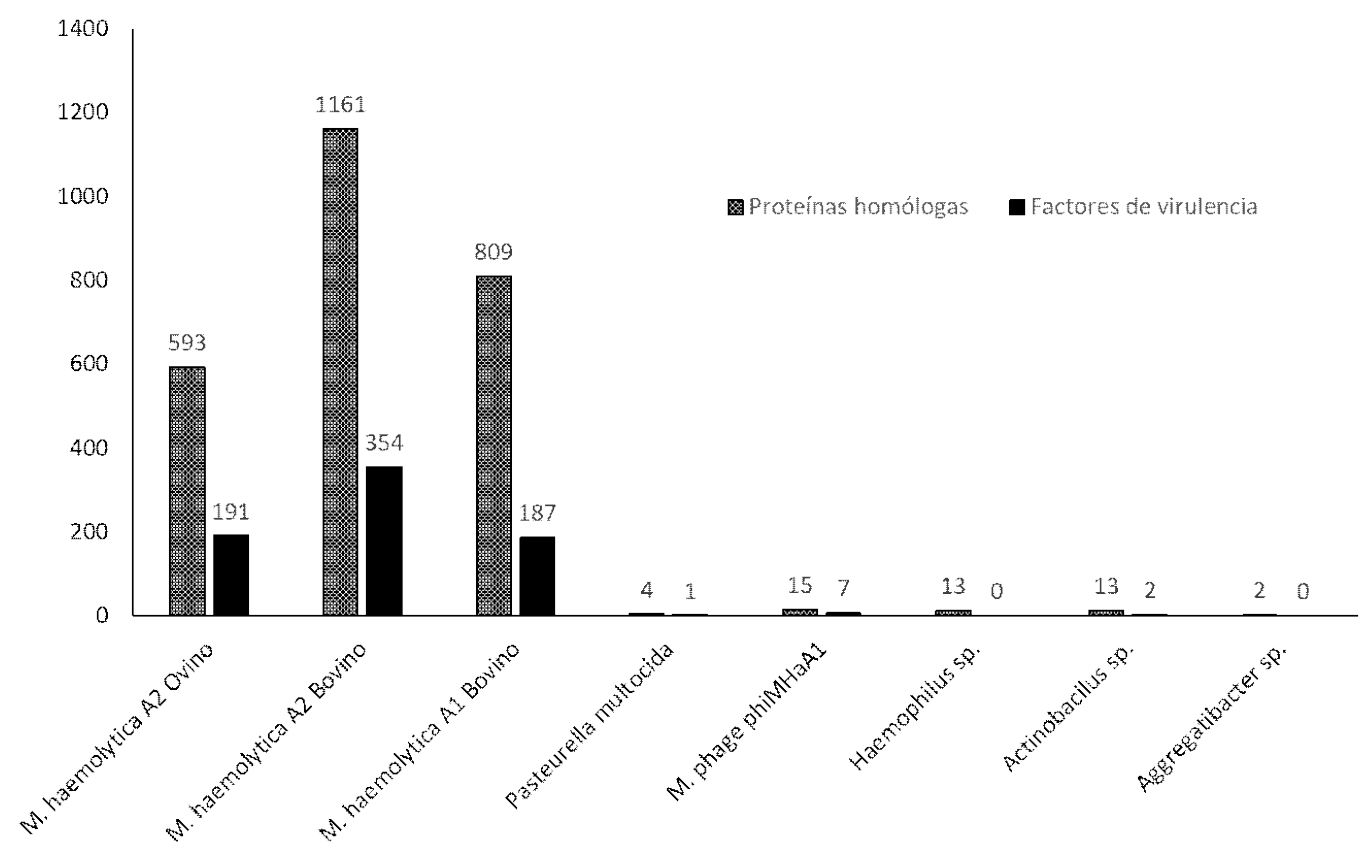

Figura 2. Distribución de proteínas homólogas y factores de virulencia identificados en el genoma de Mannheimia haemolytica serotipo $\mathrm{A}_{2}$ Ovino, en una búsqueda contra la base de datos $\mathrm{nr}(\mathrm{NCBI})$ y MvirDB, respectivamente. En ambos casos se consideró un evalue $\leq 1 \mathrm{e}-10$

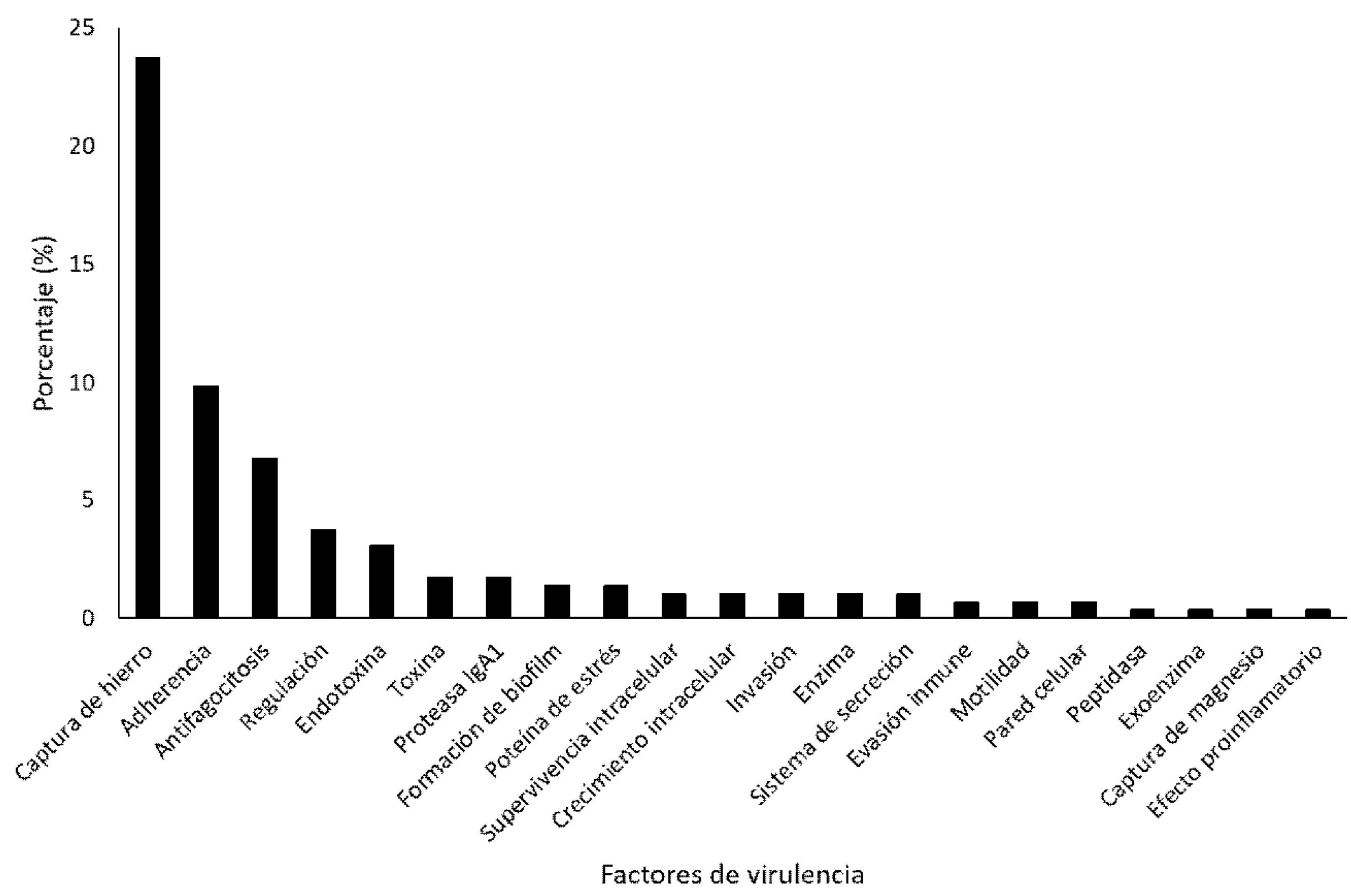

Figura 3. Porcentaje de factores de virulencia de Mannheimia haemolytica serotipo $\mathrm{A}_{2}$ Ovino, obtenidos durante la búsqueda contra la base de datos de factores de virulencia (VFDB) 


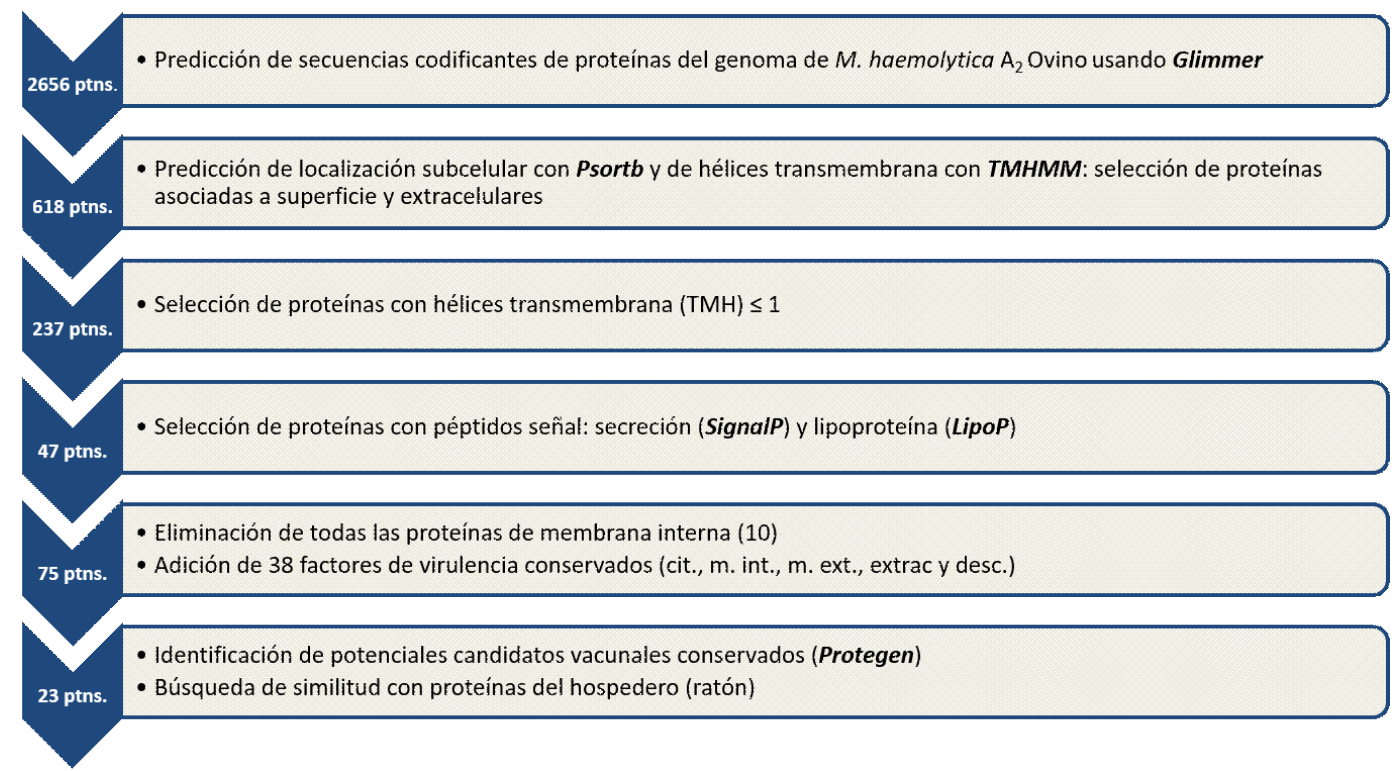

Figura 4. Diagrama de flujo de los criterios basados en el enfoque genómico de la vacunología reversa, utilizado en la identificación de factores de virulencia de $M$. haemolytica serotipo $\mathrm{A}_{2}$ Ovino como potenciales candidatos vacunales contra neumonía. cit.: citoplasmática; desc.: desconocida; $\mathrm{m}$. int.: membrana interna; m. ext.: membrana externa; extrac.: extracelular

nas citoplasmáticas o de membrana interna, no representan buenos blancos para el desarrollo de vacunas debido a que no tienen un contacto cercano a las células B del hospedero. Sin embargo, para la identificación de potenciales candidatos vacunales, donde la respuesta de células $\mathrm{T}$ es crítica, la localización subcelular no es un problema, ya que una respuesta de células $T$ puede ser dirigidas a cualquier proteína objetivo (He et al., 2010).

Debido a lo anteriormente expuesto, posterior al screening secundario, se adicionaron manualmente y en base a literatura publicada, 38 factores de virulencia potenciales, incluyendo proteínas de localización citoplasmática, de membrana externa y extracelular. Además, fueron incluidas proteínas hipotéticas de localización desconocida con el fin de identificar nuevos antígenos. No obstante, ninguna proteína citoplásmática resultó incluida en la lista final de candidatos vacunales, lo que demuestra la eficacia del pipeline de predicción empleado. Asimismo, proteínas con múltiples regiones transmembrana fueron ignoradas, ya que, en estudios similares, 250 de 600 candidatos vacunales de $N$. meningitidis $\mathrm{B}$ no pudieron ser clonados y expresados por la presencia de más de una hélice transmembrana (Pizza et al., 2000).

Se seleccionaron factores de virulencia conservados en diferentes serotipos virulentos de la misma especie y en especies relacionadas, ya que estos ofrecen una protección cruzada y «universal» (Tettelin et al., 2005). La selección final de los potenciales candidatos vacunales conservados contra la pasteurelosis neumónica fue apoyada por una base de datos de antígenos inmunoprotectivos, experimentalmente verificados (Protegen), y por la remoción de proteínas menores de 150 aminoácidos y mayores de 1000 aminoácidos. Este criterio fue incluido para asegurar que 
los candidatos puedan ser clonados y expresados exitosamente en estudios posteriores. La búsqueda de homología con proteínas de ratón fue realizada, ya que un candidato vacunal con similitud de secuencias en el hospedero es probablemente poco inmunógeno debido al mimetismo del epítope o a la producción de autoinmunidad en el hospedero (Wilson et al., 2000). Un gran porcentaje de los candidatos vacunales seleccionados estuvieron localizados en la membrana externa $(82 \%)$. Estos resultados coinciden con el alto porcentaje de proteínas de membrana externa identificadas en el genoma de $M$. haemolytica A2 Ovino, incluyendo proteínas de captación de hierro y de adherencia (Figura 3). Además, todos los antígenos seleccionados presentaron alta similitud con proteínas homólogas de los tres serotipos virulentos de M. haemolytica (e» $99 \%$ ) y de otras especies patógenas asociadas a neumonía, donde la capacidad inmunoprotectiva de la mayoría ellos ha sido verificada experimentalmente (Sutherland et al., 1989; Potter et al., 1999; Confer et al., 2003; Davies y Lee, 2004).

Entre los potenciales candidatos vacunales seleccionados destaca la leucotoxina A (orf00438), considerado el principal factor de virulencia de M. haemolytica, causante de la lisis de macrófagos y neutrófilos en bovinos y ovinos (Clinkenbeard et al., 1989). Además, existen diversos estudios que han demostrado su capacidad inmunoprotectora (Conlon et al., 1991). En el presente estudio, la leucotoxina A demostró ser homóloga con la proteína ApxiIA de Actinobacillus pleuropneumoniae (e-value $=0 ; 65 \%$ identidad y $80 \%$ de similitud), un patógeno de la familia Pasteurellaceae causante de la pleuroneumonía porcina. ApxIIA, al igual que la leucotoxina $\mathrm{A}$, es una exotoxina de la familia RTX, y su importancia como candidato vacunal ha sido demostrado en recientes estudios (Seo et al., 2013).

Asimismo, se seleccionaron proteínas de unión a transferrina tipo B (orf02261) y A (orf02262), que son clasificadas como pro- teínas de membrana externa reguladas por hierro (IROMPs), las cuales han demostrado ser inmunogénicas en bovinos $y$, en combinación con sobrenadantes de cultivo, incluyendo leucotoxina, lipopolisacárido y polisacáridos capsulares, resultaron ser inmunoprotectivos contra la infección experimental por $M$. haemolytica serotipo A1 (Sreevatsan et al., 1996). En general, estas proteínas son potenciales candidatos vacunales, ya que la adquisición de hierro es imprescindible para $M$. haemoltyica durante el estadio temprano de la infección; así como para la producción de leucotoxina y otros factores de virulencia (Ogunnariwo et al., 1997).

Se identificó una proteína de membrana externa modificable al calor (orf01914), también conocida como PomA. Esta proteína muestra gran similitud a las proteínas OmpA2 de Haemophilus ducreyi (73\%) y OmpA (P5) de Haemophilus influenzae (68\%). OmpA es una proteína altamente conservada en bacterias Gram negativas y está involucrada en la adherencia a tejidos de hospederos en varios patógenos asociados a neumonía, incluyendo $H$. influenzae y $P$. multocida (Davies y Lee, 2004). Por lo tanto, PomA puede jugar un rol en la adherencia y la colonización de $M$. haemolytica al tracto respiratorio de su hospedero.

Finalmente, entre las proteínas seleccionadas figuran dos proteínas hipotéticas COI_0728 y MHA_0690 (orf00685 y orf01126, respectivamente). Comparación y análisis de dominios demostraron que el producto orf00685 probablemente sea una lipoproteína y potencial candidato vacunal. En su lugar, la proteína hipotética MHA_0690 (orf01126), muestra una similitud del $49 \%$ con casi toda la secuencia corta de la proteína 26 de membrana externa de $H$. influenza. A esto hay que agregar que la producción de esta proteína ha demostrado ser efectiva en incrementar el aclaramiento pulmonar y en inducir títulos altos de anticuerpos mucosal y sistémico; además de una respuesta inmune celular (El-Adhami et al., 1999). Por lo tanto, la proteína hipotética MHA_0690 
(orf01126), podría ser un potencial antígeno inmunoprotectivo.

\section{Conclusiones}

- Se identificaron 23 potenciales antígenos inmunoprotectivos conservados entre los serotipos virulentos de $M$. haemolytica, utilizando el genoma de $M$. haemolytica A2 Ovino, mediante el enfoque genómico de la vacunología reversa.

- La mayoría de los antígenos identificados están asociados a la membrana externa y tienen alta similitud con factores de virulencia de otros patógenos de la familia Pasteurellaceae involucrados en procesos infecciosos neumónicos, por lo que resultan candidatos vacunales prometedores para el desarrollo futuro de vacunas recombinantes, efectivas y universales, con el fin de prevenir y controlar la pasteurelosis neumónica en alpacas.

\section{Agradecimientos}

Este estudio fue financiado por el Programa Nacional de Innovación para la Competitividad y Productividad - Innóvate Perú del Proyecto Contrato N. ${ }^{\circ} 133$-FINCyTIB-2013 «Vacunología reversa: desarrollo de una vacuna de nueva generación para el control y/o prevención de la neumonía pasteurelósica en alpacas».

\section{Literatura Citada}

1. Altschul SF, Madden TL, Schäffer AA, Zhang J, Zhang Z, Miller W, Lipman DJ. 1997. Gapped BLAST and PSIBLAST: a new generation of protein database search programs. Nucleic Acids Res 25: 3389-3402.

2. Angen O, Quirie M, Donachie W, Bisgaard M. 1999. Investigations on the species specificity of Mannheimia (Pasteurella) haemolytica serotyping.
Vet Microbiol 65: 283-290. doi: 10.1016/ S0378-1135(98)00304-6

3. Bambini S, Rappuoli R. 2009. The use of genomics in microbial vaccine development. Drug Discov Today 14: 252-260. doi: 10.1016/j.drudis.2008.12.007

4. Chen L, Zheng D, Liu B, Yang J, Jin Q. 2016. VFDB 2016: hierarchical and refined dataset for big data analysis - 10 years on. Nucleic Acids Res 44: D694D697. doi: 10.1093/nar/gkv1239

5. Clinkenbeard KD, Mosier DA, Confer $A W .1989$. Effects of Pasteurella haemolytica leukotoxin on isolated bovine neutrophils. Toxicon 27: 797-804. doi: 10.1016/0041-0101(89)90047-0

6. Confer AW, Ayalew S, Panciera RJ, Montelongo $M$, Whitworth LS, Hammer JD. 2003. Immunogenicity of recombinant Mannheimia haemolytica serotype 1 outer membrane protein PlpE and augmentation of a commercial vaccine. Vaccine 21: 2821-2829. doi: 10.1128/CVI.05332-11

7. Conlon JA, Shewen PE, Lo RY. 1991. Efficacy of recombinant leukotoxin in protection against pneumonic challenge with live Pasteurella haemolytica A1. Infect Immun 59: 587-591.

8. Davies RL, Arkinsaw S, Selander RK. 1997. Evolutionary genetics of Pasteurella haemolytica isolates recovered from cattle and sheep. Infect Immun 65: 3585-3593.

9. Davies RL, Baillie S. 2003. Cytotoxic activity of Mannheimia haemolytica strains in relation to diversity of the leukotoxin structural gene $1 \mathrm{ktA}$. Vet Microbiol 92: 263-279.

10. Davies RL, Lee I. 2004. Sequence diversity and molecular evolution of the heat-modifiable outer membrane protein gene (ompA) of Mannheimia (Pasteurella) haemolytica, Mannheimia glucosida, and Pasteurella trehalosi. J Bacteriol 186: 5741-5752. doi: 10.1128/ JB.186.17.5741-5752.2004 


\section{Davies RL, Whittam TS, Selander RK.} 2001. Sequence diversity and molecular evolution of the leukotoxin (lktA) gene in bovine and ovine strains of Mannheimia (Pasteurella) haemolytica. J Bacteriol 183: 1394-1404. doi: 10.1128/JB.183.4.1394-1404.2001

12. Delcher AL, Bratke KA, Powers EC, Salzberg $S L$. 2007. Identifying bacterial genes and endosymbiont DNA with glimmer. Bioinformatics 23: 673-679. doi: 10.1093/bioinformatics/btm009

13. El-Adhami W, Kyd JM, Bastin DA, Cripps $A W$. 1999. Characterization of the gene encoding a 26-kilodalton protein (OMP26) from nontypeable Haemophilus influenzae and immune responses to the recombinant protein. Infect Immun 67: 1935-1942.

14. Ewers C, Lubke-Becker A, Wieler LH. 2004. Mannheimia haemolytica and the pathogenesis of enzootic bronchopneumonia. Berlin Munch Tierarztl Wochenschr 117: 97-115.

15. Gamberini M, Gómez RM, Atzingen MV, Martins EA, Vasconcellos SA, Romero EC, et al. 2005. Wholegenome analysis of Leptospira interrogans to identify potential vaccine candidates against leptospirosis. FEMS Microbiol Lett 244: 305-313. doi: 10.1016/ j.femsle.2005.02.004

16. Gioia J, Qin $X$, Jiang $H$, Clinkenbeard K, Lo R, Liu Y, Fox GE, et al. 2006. The genome sequence of Mannheimia haemolytica A1: insights into virulence, natural competence, and Pasteurellaceae phylogeny. J Bacteriol 188: 7257-7266. doi: 10.1128/JB.00675-06

17. Gonzalez JM, Lacasta D, Ferrer LM, Figueras L, Abadie G, Heras de las M. 2013. Mannheimia haemolytica and Bibersteinia trehalosi serotypes isolated from lambs with ovine respiratory complex in Spain. J Hellenic Vet Med Soc 64: 177-182.

18. He Y, Xiang Z, Mobley HL. 2010. Vaxign: the first web-based vaccine design program for reverse vaccinology and applications for vaccine development. J Biomed Biotechnol 2010: 297505. doi: 10.1155/2010/297505

19. Huang X, Madan A. 1999. CAP3: a DNA sequence assembly program. Genome Res 9: 868-877. doi: 10.1101/ gr.9.9.868

20. Juncker AS, Willenbrock $H$, Von Heijne G, Brunak S, Nielsen H, Krogh A. 2003. Prediction of lipoprotein signal peptides in Gram-negative bacteria. Protein Sci 12: 1652-1662. doi: 10.1110/ ps.0303703

21. Kehrenberg C, Schulze-Tanzil G, Martel JL, Chaslus-Dancla E, Schwarz S. 2001. Antimicrobial resistance in Pasteurella and Mannheimia: epidemiology and genetic basis. Vet Res 32: 323-339. doi: 10.1051/ vetres:2001128

22. Krogh A, Larsson B, von Heijne $G$, Sonnhammer EL. 2001. Predicting transmembrane protein topology with a hidden Markov model: application to complete genomes. J Mol Biol 305: 567580. doi: $10.1006 / \mathrm{jmbi} .2000 .4315$

23. Lacasta D, Ferrer LM, Ramos JJ, González JM, Ortín A, Fthenakis GC. 2015. Vaccination schedules in small ruminant farms. Vet Microbiol 181: 3446. doi: 10.1016/j.vetmic.2015.07.018

24. Lawrence PK, Kittichotirat W, Bumgarner RE, McDermott JE, Herndon DR, Knowles DP, Srikumaran S. 2009. Genome sequences of Mannheimia haemolytica serotype A2: ovine and bovine isolates. J Bacteriol 192: 1167-1168. doi: 10.1128/JB.01527-09

25. Lawrence PK, Kittichotirat $W$, McDermott JE, Bumgarner RE. 2010. A three-way comparative genomic analysis of Mannheimia haemolytica isolates. BMC Genomics 11: 535. doi: 10.1186/1471-2164-11-535

26. Mohamed RA, Abdelsalam EB. 2008. A review on pneumonic pasteurellosis (respiratory mannheimiosis) with emphasis on pathogenesis, virulence mechanisms and predisposing factors. Bulg J Vet Med 11: 139-160. 
27. Ogunnariwo JA, Woo TK, Lo RY, Gonzalez GC, Schryvers AB. 1997. Characterization of the Pasteurella haemolytica transferrin receptor genes and the recombinant receptor proteins. Microb Pathog 23: 273-284. doi: 10.1006/ mpat.1997.0156

28. Petersen TN, Brunak S, von Heijne G, Nielsen H. 2011. SignalP 4.0: discriminating signal peptides from transmembrane regions. Nat Methods 8: 785-786. doi: 10.1038/nmeth. 1701

29. Pizza M, Scarlato V, Masignani V, Giuliani MM, Arico B, Comanducci M, Jennings GT, et al. 2000. Identification of vaccine candidates against serogroup B meningococcus by whole-genome sequencing. Science 287: 1816-1820. doi: 10.1126/science.287.5459.1816

30. Potter AA, Schryvers AB, Ogunnariwo JA, Hutchins WA, Lo RY, Watts T. 1999. Protective capacity of the Pasteurella haemolytica transferrinbinding proteins TbpA and $\mathrm{TbpB}$ in cattle. Microb Pathog 27: 197-206. doi: 10.1006/ mpat.1999.0297

31. Rice P, Longden I, Bleasby A. 2000. EMBOSS: the European Molecular Biology Open Software Suite. Trends Genetics 16: 276-277. doi: 10.1016/ S0168-9525(00)02024-2

32. Rosadio R, Cirilo E, Manchego A, Rivera $H .2011$. Respiratory syncytial and parainfluenza type 3 viruses coexisting with Pasteurella multocida and Mannheimia hemolytica in acute pneumonias of neonatal alpacas. Small Ruminant Res 97: 110-116. doi: 10.1016/ j.smallrumres.2011.02.001

33. Saadati M, Gibbs H, Parton R, Coote $J$. 1997. Characterisation of the leukotoxin produced by different strains of Pasteurella haemolytica. J Meed Microbiol 46: 276-284. doi: 10.1099/ 00222615-46-4-276

34. Seo KW, Kim SH, Park J, Son Y, Yoo HS, Lee KY, Jang YS. 2013. Nasal immunization with major epitopecontaining ApxIIA toxin fragment indu- ces protective immunity against challenge infection with Actinobacillus pleuropneumoniae in a murine model. Vet Immunol Immunopathol 151: 102-112. doi: 10.1016/j.vetimm.2012.10.011

35. Sreevatsan S, Ames TR, Werdin RE, Yoo HS, Maheswaran SK. 1996. Evaluation of three experimental subunit vaccines against pneumonic pasteurellosis in cattle. Vaccine 14: 147-154. doi: 10.1016/0264-410X(95)00138-Q

36. Stajich JE, Block D, Boulez K, Brenner SE, Chervitz SA, Dagdigian $C$, Fuellen G, et al. 2002. The bioperl toolkit: perl modules for the life sciences. Genome Res 12: 1611-1618. doi: 10.1101/ gr.361602

37. Sutherland AD, Donachie W, Jones GE, Quirie M. 1989. A crude cytotoxin vaccine protects sheep against experimental Pasteurella haemolytica serotype A2 infection. Vet Microbiol 19: 175-181. doi: 10.1016/0378-1135(89)90082-5

38. Tettelin H, Masignani V, Cieslewicz MJ, Donati C, Medini D, Ward NL, Angiuoli SV, et al. 2005. Genome analysis of multiple pathogenic isolates of Streptococcus agalactiae: implications for the microbial «pan-genome». Proc Natl Acad Sci USA 102: 1395013955. doi: 10.1073/pnas.0506758102

39. Wilson C, Tiwana H., Ebringer A. 2000. Molecular mimicry between HLA-DR alleles associated with rheumatoid arthritis and Proteus mirabilis as the aetiological basis for autoimmunity. Microbes Infect 2: 1489-1496. doi: 10.1016/S12864579(00)-01303-4

40. Yang B, Sayers $S$, Xiang $Z, H e, Y$. 2011. Protegen: a web-based protective antigen database and analysis system. Nucleic Acids Res 39: D1073-D1078. doi: $10.1093 / \mathrm{nar} / \mathrm{gkq} 944$

41. Yu NY, Wagner JR, Laird MR, Melli G, Rey S, Lo R, Dao P, et al. 2010. PSORTb 3.0: improved protein subcellular localization prediction with refined localization subcategories and predictive capabilities for all prokaryotes. 
Bioinformatics 26: 1608-1615. doi: 10.1093/bioinformatics/btq249

42. Zecchinon L, Fett T, Desmecht D. 2005. How Mannheimia haemolytica defeats host defence through a kiss of death mechanism. Vet Res 36: 133-156. doi: 10.1051/vetres:2004065
43. Zhou CE, Smith J, Lam M, Zemla A, Dyer MD, Slezak T. 2007. MvirDBa microbial database of protein toxins, virulence factors and antibiotic resistance genes for bio-defence applications. Nucleic Acids Res 35: D391-D394. doi: 10.1093/nar/gk1791 